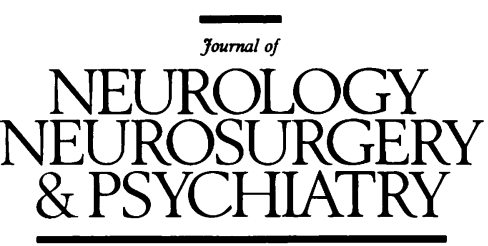

\title{
Editorial
}

\section{Cerebral venous thrombosis: developments in imaging and treatment}

Thrombosis of the cerebral venous system may involve the cortical veins alone, the dural sinuses alone, or both. Isolated cortical venous thrombosis is rare, most patients therefore having dural sinus thrombosis with or without cortical vein involvement. The term cerebral venous thrombosis conveniently includes all types of venous thrombosis within its umbrella, and will be used for that purpose in this article, except where sufficient information has allowed a more specific anatomical diagnosis.

The incidence of cerebral venous thrombosis remains uncertain. Previous reviews have relied on necropsy data, or, more recently, on diagnosis established by cerebral angiography. ${ }^{1}$ Despite a voluminous list of conditions considered to be associated with cerebral venous thrombosis, some $25 \%$ of cases remain of uncertain aetiology. ${ }^{2}$ The clinical range of cerebral venous thrombosis is broad, and has widened further as techniques for its diagnosis have developed. In one series, ${ }^{1}$ six patterns of presentation were recognised, and in their order of frequency were the progressive onset of signs of intracranial hypertension; the sudden onset of focal deficit mimicking an arterial stroke but with more frequent seizures; the evolution over a few days of focal deficit with or without seizures and intracranial hypertension, the same pattern evolving over weeks or months; and, least commonly, the sudden onset of headache simulating subarachnoid haemorrhage or a picture suggesting a transient ischaemic attack.

Angiography remains the gold standard for diagnosis ${ }^{3}$ with a digital intravenous subtraction technique replacing conventional angiography for diagnostic purposes in patients with suspected abnormality of the larger intracranial veins and sinuses. Increasingly, data have emerged on the role of CT and MRI in diagnosis to the point where non-invasive procedures allow accurate diagnosis in most cases. This review will not consider cavernous sinus thrombosis, nor thrombosis of the galenic system or the cerebellar veins.

\section{Imaging}

Besides CT and MRI, other imaging techniques have been evaluated for their role in the detection of venous thrombosis, particularly where it affects the superior sagittal sinus. Ultrasonography has successfully detected superior sagittal sinus thrombosis in infants. ${ }^{5}$ With indium-111 labelled platelets, superior sagittal sinus thrombosis was detected in a patient in whom CT had failed to show evidence of thrombus. The diagnosis was confirmed by formal angiography. ${ }^{6}$ Technetium-labelled red cells have been used in a similar fashion, and evaluated in a group of 19 patients suspected of having superior sagittal sinus thrombosis. ${ }^{7}$ The sensitivity of technetium-labelled red cell scintigraphy was tested in a group of 12 patients in whom the diagnosis was considered sound on clinical and CT criteria. On the basis of a single false positive result the authors claimed a sensitivity of $100 \%$ and a specificity of $86 \%$ for the procedure. The widespread availability of CT and MRI suggests that these other techniques will not gain favour in the evaluation of suspected cerebral venous thrombosis.

\section{Computed tomography}

A substantial scientific literature has accumulated on the role of CT in establishing the diagnosis of cerebral venous thrombosis. Changes described include areas of hyperdensity and hypodensity, cerebral oedema, hydrocephalus, the cord sign, and delta sign, along with abnormal enhancement after contrast injection. An early review of 11 cases, diagnosed either by angiography or from pathological data, included examples of all the changes. ${ }^{8}$ The cord sign refers to a thrombosed vein, present on precontrast views, whereas the delta sign refers to an empty triangular shape visible within the posterior aspect of the superior sagittal sinus after contrast injection. The same area may be seen as abnormally dense on precontrast films. Variation in the window setting may be necessary to define the delta sign more accurately. ${ }^{9}$ If the appearance is doubtful, thin (less than $5 \mathrm{~mm}$ ) sections should be obtained and the sign only accepted if present on several sections. ${ }^{10}$ High splitting of the superior sagittal sinus can mimic a delta $\operatorname{sign}^{11}$ as can an adjacent epidural abscess. An analysis of the sign on postcontrast scans showed its presence in $29 \%$ of 76 cases of superior sagittal thrombosis or superior sagittal related intracranial sinovenous occlusive disease reported from the medical literature. ${ }^{10}$ In the same review, only $3.6 \%$ of the scans were normal, although rather higher figures have been reported in individual series. From this overview of CT changes in 24 series, cerebral haemorrhage was encountered unilaterally in $8.5 \%$ of cases, bilaterally in $6 \%$, with cerebral oedema in $8 \%$ and compressed ventricles in $14 \%$. More recent papers have claimed a higher figure for the delta sign on contrast enhanced CT, reaching $70 \%$ in one series of 14 patients. ${ }^{12}$ The variation in the reporting 
of the sign is partly explained by the absence of postcontrast scans in some early studies, and also by the delay in its appearance in some cases and its subsequent resolution. In most cases the sign has taken several days or weeks to resolve, although in a single case report of dubious value, it had resolved within a day, admittedly in a patient in whom angiography within 24 hours of admission had failed to show evidence of superior sagittal thrombosis. ${ }^{13}$ Other CT findings in cerebral venous thrombosis are rare, although in one patient, prominent dilatation of the deep transcerebral medullary veins on postcontrast CT was found in the absence of either a delta or cord sign, and in the absence of tentorial or gyral enhancement. ${ }^{14}$ Recent publications have reinforced the need for thin section multiplanar reconstruction or direct coronal scanning when evaluating equivocal CT abnormalities. ${ }^{15}$

Normal scans are more likely where the thrombotic process has been confined to the deep venous system, including the vein of Galen and the straight sinus, ${ }^{10}$ and also seem more common if the patient has presented with signs of intracranial hypertension alone. ${ }^{12}$ Patients who have both an empty delta sign and evidence of haemorrhagic infarction on CT seem to have a worse prognosis. ${ }^{10}$

\section{Magnetic resonance imaging}

Magnetic resonance imaging is now established as the imaging technique of choice in the immediate evaluation of cerebral venous thrombosis. Most of the publications on this technique have concentrated on how differing imaging sequences allow evaluation of thrombus formation and the distinction of intraluminal clot from artefact.

With spin echo sequences, low blood flow velocity produces a high intensity signal that may be confused with the signal changes produced in a thrombus. ${ }^{16}$ Serial scanning has determined the signal characteristics of an evolving venous thrombosis. Initially the normal flow void is absent in any imaging sequence. The thrombus is isointense on $\mathrm{T} 1$ and hypointense on $\mathrm{T} 2$ weighted images. Subsequently the thrombus becomes hyperintense on both T1 and T2 images. After some two weeks, flow void reappears consequent to vascular recanalisation. Various methods have been developed to reduce or eliminate the possibility of flow related artefacts. Spin echo techniques that eliminate flow related enhancement can be used to differentiate thrombus from flow artefact. ${ }^{17}$ Magnetic resonance phase imaging has also been advocated as a suitable technique for imaging blood flow $^{18}$ and is considered to be more reliable than gradient echo imaging. It can be performed simultaneously with the routine spin echo sequence and seems to correlate well with conventional angiography. ${ }^{19}$ Two recent papers have further considered the changing pattern of thrombus with time as shown by spin echo techniques. ${ }^{2021}$ In one, 23 patients were described of whom 18 had had formal angiography. ${ }^{21}$ Four phases, distinguished by their MRI characteristics, were encountered. In phase 1 , from days 1 to 5 after the onset of symptoms, the thrombus was hypointense on $\mathrm{T} 2$ and isointense on $\mathrm{T} 1$ weighted images. From days 6 to 15, hyperintense signals emerged on both sequences. From day 16 to the end of the third month, the signal declined in all sequences and flow signals increased on three dimensional angiography. After four months, some patients showed a persistent isointense intraluminal signal with absent flow signal indicating permanent occlusion. In five other patients, the results suggested complete recanalisation. Only a proportion of the patients in this study were sequenced through the whole period and all had been anticoagulated although not necessarily immediately after the onset of symptoms. The authors concluded that a certain diagnosis could be made in the first two weeks from MRI techniques alone, whereas longer standing thrombus would require intra-arterial arteriography for confident diagnosis. The authors also suggested that the diagnosis of occlusion of cortical veins rather than the dural sinuses demanded conventional angiography. In another recent report signal abnormalities on MRI were absent in two out of 53 cases of cerebral venous thrombosis. ${ }^{21}$

\section{Magnetic resonance angiography}

Both the arterial and venous systems of the brain can be visualised by appropriate MR techniques. ${ }^{22}$ Subsequently the method has been applied to the diagnosis of cerebral venous thrombosis. ${ }^{23-25}$ At present, the major veins and sinuses, such as the superior sagittal, straight and lateral sinuses, the internal cerebral veins, and the veins of Galen, can be reliably imaged. ${ }^{25}$ Account has to be taken of anatomical variation, as it does with conventional angiography. Absence of a vein or sinus can be attributed to thrombus only if the clot can be visualised on spin echo images at the relevant site. Increasingly the technique, along with conventional MRI, is becoming the initial investigation of choice in cerebral venous thrombosis with conventional angiography reserved for those cases with a clinically suspicious history but a negative study. ${ }^{3}$

\section{Management}

Recent developments in management have depended on more reliable data regarding the role of anticoagulant treatment, and information on the value of direct thrombolytic treatment.

A study combining both retrospective and prospective elements has considerably influenced opinion on the role of anticoagulant treatment. ${ }^{26}$ Over a two year period, a randomised, single blind, placebo controlled trial was performed of dose adjusted intravenous heparin in 28 patients with angiographically established sinus venous thrombosis. Clinical detail on the patients is sparse. They were entered into the study about one month after the onset of symptoms. Patients with sepsis, tumours, or cavernous sinus thrombosis were excluded. After further exclusions, 20 patients remained for randomisation. The target partial thromboplastin time in the treatment group was 80-100 seconds. The treating physician was unblinded although daily assessments were performed by a blinded physician. A rating scale of severity of symptoms and disability was developed for use in the study. After three months, eight patients in the heparin group had completely recovered and two had slight neurological deficits. In the control group one recovered completely, six had neurological deficits, and three died. On repeat CT, no fresh intracranial haemorrhages appeared in the heparin group. Concomitant steroid treatment was given to three of the control but none of the heparin group. Although numbers were small and the design not double blinded, the results are sufficiently persuasive to recommend heparin treatment in cases of cerebral venous thrombosis. Curiously, the authors failed to indicate for how long heparin was used in this study, nor did they make recommendations about the duration of any subsequent Warfarin treatment. Perhaps more curiously, although the prospective trial was completed in 1984, its publication was delayed for seven years.

On the basis of this trial, it would seem reasonable to recommend intravenous heparin for patients with 
cerebral venous thrombosis, although it is impossible from study of the paper to know whether patients with intracranial hypertension alone were entered. The presence of an intracerebral haematoma at initial scanning does not seem to contraindicate heparin treatment.

Direct thrombolytic treatment for superior sagittal sinus thrombosis has been successfully performed in infants. ${ }^{27}$ In a case report of an adult with thrombosis of the superior sagittal, straight, and transverse sinuses, infusion of urokinase into the confluence of the sinuses and subsequently into the superior sagittal sinus led to partial clot lysis (determined angiographically) and a good clinical outcome. ${ }^{28}$ Further studies totalling 12 patients with either superior sagittal, transverse, or sigmoid sinus thrombosis have been reported. ${ }^{29}{ }^{30}$ In the first study, ${ }^{29}$ urokinase infusions were performed within about 48 hours of onset of symptoms in at least four of the five cases, two of whom had deteriorated on heparin. In the second study, ${ }^{30}$ comprising seven patients, symptoms had been present from one to 24 weeks before treatment. In two patients, progressive lethargy was considered a part of the disorder. All the patients had had prior anticoagulant treatments, and heparin was continued during the infusions, which averaged 163 hours in duration. Interpretation of the value of treatment is made difficult in this study by the fact that one patient had subsequent surgery to a transverse sinus stenosis, one required repair of a dural arteriovenous fistula, and a third was eventually discovered to have an astrocytoma. None of the 12 patients in these two series, incidentally, had evidence of intracranial haemorrhage at the onset of treatment. No major complications were found either from the infusions or from the procedure of direct dural sinus venography.

\section{Conclusions}

The incidence of cerebral venous thrombosis is almost certainly underestimated. Conventional angiography has been the gold standard for diagnosis but is rapidly being replaced by conventional MRI coupled with MR angiography. Results of CT are usually abnormal in this condition, but specific abnormalities-for example, the delta sign-have been found in a minority of patients in most series, and even then they are not wholly diagnostic. Magnetic resonance imaging is of particular value in the diagnosis in patients with a history of less than two weeks. A confusing plethora of imaging techniques is described for separating genuine thrombus formation from artefact. Magnetic resonance angiography readily identifies the major sinuses but cannot yet identify the cortical veins with the same accuracy as conventional angiography.

Until recently the mainstay of treatment has been intravenous heparin, although there is no consensus about the duration of treatment, nor whether it should be followed by oral anticoagulant treatment. Direct instillation of thrombolytic agents into the affected sinus is technically feasible and has produced a successful outcome. The indications for direct thrombolytic treatment as opposed to heparinisation, the dosage and mode of infusion, and the exact morbidity of the procedure and of the treatment are undecided. A controlled trial of thrombolytic treatment $v$ heparin in life threatening sinus venous thrombosis is called for.

G D PERKIN
Deiences Centre, Charing Cross Hospital, Fulham Palace Road London W6 8RF, UK

1 Bousser MG, Chiras J, Bories J, Castaigne P. Cerebral venous thrombosis-a review of 38 cases. Stroke 1985;16:199-213.

2 Bousser MG. Thromboses veineuses cerebrales. A propos de 76 cas. f Mal Vasc 1991;16:249-55.

3 Chelly D, Levy C, Ameri A, Brunereau L. Imagerie des thrombophlebites cerebrales. Ann Radiol (Paris) 1994;37:108-17.

4 Little JR, Furlan AJ, Modic MT, Weinstein MA. Digital subtraction angiography in cerebrovascular disease. Stroke 1982;13:557-66.

5 Edwards MK, Kuharik MA, Cohen MD. Sonographic demonstration of cerebral sinus thrombosis. AfNR Am f Neuroradiol 1987;8:1153-5.

6 Bridgers SL, Strausse E, Smith EO, Reed D, Ezekowitz MD Demonstration of superior sagittal sinus thrombosis by indium-111 Demonstration of superior sagittal sinus thrombosis
platelet scintigraphy. Arch Neurol 1986;43:1079-81.

7 Front D, Israel O, Even-Sapir E, Feinsud M. Superior sagittal sinus thrombosis: assessment with Tc-99m labeled red blood cells. Radiology
throm 1986;158:453-6.

8 Buonanno FS, Moody DM, Ball MR, Laster DW. Computed cranial tomographic findings in cerebral sinovenous occlusion. $\mathcal{f}$ Comput Assist Tomogr 1978;2:281-90.

9 Brant-Zawadzki, Chang GY, McCarty GE. Computed tomography in dural sinus thrombosis. Arch Neurol 1982;39:446-7.

10 Virapongse C, Cazenave C, Quisling R, Sarwar M, Hunter S. The empty delta sign: frequency and significance in 76 cases of dural sinus thrombosis. Radiology 1987;162:779-85.

11 Rao KCVG, Knipp HC, Wagner EJ. Computed tomographic findings in cerebral sinus and venous thrombosis. Radiology 1981;140:391-8.

12 Thron A, Wessel K, Linden D, Schroth G, Dichgans J. Superior sagittal sinus thrombosis: neuroradiological evaluation and clinical findings. $f$ Neurol 1986;233:283-8.

13 Matsumura A, Shinohara A, Komatsu Y, Hashikawa M, Nose T Vanishing empty delta sign in cerebral venous thrombosis. AfNR Am $\dot{\mathcal{F}}$ Neuroradiol 1988;9:1239-40.

14 Anderson SC, Shah CP, Murtagh FR. Congested deep subcortical veins as a sign of dural venous thrombosis: $M R$ and $C T$ correlations. f Comput Assist Tomogr 1987;11:1059-61.

15 Goldberg AL, Rosenbaum AE, Wang H, Kim WS, Lewis Van L, Hanley DF. Computed tomography of dural sinus thrombosis. $\mathcal{F}$ Comput Assis Tomogr 1986;10:16-20.

16 Macchi PJ, Grossman RI, Gomori JM, Goldberg HI, Zimmerman RA, Bilaniuk LT. High field MR imaging of cerebral venous thrombosis. $\mathcal{f}$ Comput Assist Tomogr 1986;10:10-5.

17 Sze G, Simmons B, Krol G, Walker R, Zimmerman RD, Deck MDF Dural sinus thrombosis: verification with spin-echo techniques. AfNR Am 7 Neuroradiol 1988;9:679-86.

18 Nadel L, Braun IF, Kraft KA, Jensen ME, Laine FJ. MRI of intracranial sinovenous thrombosis: the role of phase imaging. Magn Reson Imaging 1990;8:315-20.

19 Nadel L, Braun IF, Kraft KA, Fatouros PP, Laine FJ. Intracranial vascular abnormalities: value of MR phase imaging to distinguish thrombus from flowing blood. AfNR Am f Neuroradiol 1990;11:1133-40.

20 Isensee $\mathrm{Ch}$, Reul J, Thron A. Magnetic resonance imaging of thrombosed dural sinuses. Stroke 1994;25:29-34.

21 Dormont D, Anxionnat R, Evrard S, Louaille C, Chiras J, Marsault C. MRI in cerebral venous thrombosis. $\mathcal{f}$ Neuroradiol 1994;21:81-99.

22 Dumoulin CL, Hart HR Jr. Magnetic resonance angiography. Radiology 1986;161:717-20.

23 Rippe DJ, Boyko OB, Spritzer CE, Meisler WJ, Dumoulin CL, Souza SP Heinz ER. Demonstration of dural sinus occlusion by the use of MR angiography. AfNR Am $\mathcal{F}$ Neuroradiol 1990;199-201.

24 Padayachee TS, Bingham JB, Graves MJ, Colchester ACF, Cox TCS. Dural sinus thrombosis. Diagnosis and follow-up by magnetic resonance angiography and imaging. Neuroradiology 1991;33:165-7.

25 Mattle HP, Wentz KU, Edelman RR, Wallner B, Finn JP, Barnes P, et al. Cerebral venography with MRI. Radiology 1991;178:453-8.

26 Einhaupl KM, Villringer A, Meister W, Mehraein S, Garner C, Pellkofer $M$, et al. Heparin treatment in sinus venous thrombosis. Lancet 1991; 338:597-600.

27 Higashida RT, Helmer E, Halbach VV, Hieshima GB. Direct thrombolytic therapy for superior sagittal sinus thrombosis. AfNR Am $\mathcal{f}$ Neuroradiol 1989:10:S4-6.

28 Eskridge JM, Wessbecher FW. Thrombolysis for superior sagittal sinus thrombosis. $\mathcal{f}$ Vasc Interv Radiol 1991;2:89-93.

29 Tsai FY, Higashida RT, Matouich V, Alfieri K. Acute thrombosis of the intracranial dural sinus: direct thrombolytic treatment. $A f N R A m \mathcal{F}$ Neuroradiol 1992;13:1137-41.

30 Smith TP, Higashida RT, Barnwell SL, Halbach VV, Dowd CF, Fraser $\mathrm{KW}$, et al. Treatment of dural sinus thrombosis by urokinase infusion. AfNR Am $\mathcal{F}$ Neuroradiol 1994;15:801-7. 